\title{
Multiple Myeloma: Front Line Therapy and Autologous Stem Cell Transplantation
}

\author{
Lalit Kumar \\ Department of Medical Oncology \\ Institute Rotary Cancer Hospital, All India Institute of Medical Sciences, New Delhi.
}

\begin{abstract}
Prognosis of multiple myeloma (MM) has improved during the past two decades. This has been attributed to the better understanding of the biology of disease leading to introduction of two new classes of molecules, namely immune-modulators (e.g. thalidomide, lenalidomide), and proteasome inhibitors (e.g. bortezomib), use of high dose chemotherapy and autologous stem cell transplantation (ASCT) and better supportive care. Current management of myeloma for young patients ( $\leq 65$ years) includes initial induction therapy followed by consolidation with ASCT followed by maintenance therapy with low dose thalidomide or lenalidomide or bortezomib for 1-2 years.

The choice of initial therapy for patients of MM is based upon their eligibility for ASCT which in turn is based on their age and major co-morbid conditions pertaining to cardiac and renal systems. Patients who are $\leq 65$ years of age (or 65 to 70 years) with no major co-morbid conditions are considered potential candidates for ASCT. Four cycles of induction therapy are administered; a combination of 3 drugs (bortezomib, thalidomide, and dexamethasone (BTD) or bortezomib, lenalidomide, and dexamethasone (BLD) or bortezomib, cyclophosphamide and dexamethasone (BCD) is associated with higher complete response (CR) (approx. 30-40\%) and very good partial response (VGPR) and better progression free survival (PFS). Further consolidation with ASCT results in CR rates of 50\%-70\%; patients who achieve CR, have improved event-free and overall survival. Our initial experience with 225 ASCT supports these observations.
\end{abstract}

It is now possible to individualize therapy in a given patient. For example, for patients with renal failure (present in 20-30\% of patients at diagnosis) - bortezomib, dexamethasone and/or doxorubicin combination could be an option; for patients with pre-existing peripheral neuropathy-lenalidomide and dexamethasone is preferred; for patients at high risk of venous thrombo-embolism bortezomibbased regimens can be used safely. Treatment with bortezomib or bortezomib + lenalidomide for patients with poor cytogenetics (chromosome deletion $\mathrm{t}(4 ; 14), \mathrm{t}(14 ; 16), 17 \mathrm{p}-$ ) appears to result in an outcome similar to that in patients without these abnormalities.

In conclusion, from being incurable, myeloma is now a chronic illness. Along with earlier diagnosis, improved treatment and better management of complications have resulted in longer disease control and survival with a better quality of life. Novel agents have provided an opportunity to tailor therapy in an individual patient. Further research is needed to improve outcome for patients who fail to achieve complete response, those with ISS stage III, and extra-medullary disease. Availability of oral proteasome inhibitors and monoclonal antibodies (e.g. IL-6 receptor) are likely to expand choice of agents for maintenance therapy in future.

Correspondence: Dr. Lalit Kumar, Department of Medical Oncology, Institute Rotary Cancer Hospital, All India Institute of Medical Sciences, New Delhi-110029. Email:1alitaiims@yahoo.com.

DR. V.R. KHANOLKAR ORATION delivered during NAMSCON 2015 at the All India Institute of Medical Sciences, Patna. 


\section{Introduction}

Multiple myeloma (MM)- a disease of malignant plasma cells accounts for $1 \%$ of all malignant disorders and 10-15\% of haematological malignancies. While incidence of myeloma is lower in Asia and in India compared to West, there is evidence that in metropolitan cities, incidence of $\mathrm{MM}$ is gradually rising (1). Some of key differences seen in presentation in India compared to western population include- younger age at presentation (median 55-60 years compared to 65 years), delay in diagnosis, lower proportion of asymptomatic patients (1-2\% Vs 10-20\%), higher proportion of patients with anaemia $(\mathrm{Hb}$ $<10 \mathrm{~g} / \mathrm{dL}$ ), ISS stage III (30 to $50 \%$ ), renal failure (eGFR $<40 \mathrm{ml} / \mathrm{mt}$ in $25 \%$ ) and higher proportion of patients with extra-medullary disease (10-20\%) (2). Limited data suggest that proportion of high risk cytogenetics $[17 \mathrm{p} \mathrm{del}$, $\mathrm{t}(4 ; 14), \mathrm{t}(14 ; 16)]$ is similar $(10-15 \%)$ (unpublished data).

Survival of MM patients has improved significantly during the past 2 decades. This has been attributed to novel agents based induction, autologous stem cell transplantation (ASCT) in eligible patients, and use of maintenance therapy (3). Prior to year 2000, initial therapy for myeloma patients included cytotoxic chemotherapy - melphalan and prednisolone or VAD (vincristine, adriamycin, and dexamethasone) as continuous infusion. Treatment was associated with low complete response rates $(5-15 \%)$, and short progression free and overall survival (2.5 to 3.5 years). Introduction of immunomodulators (thalidomide, lenalidomide), proteasome inhibitors (bortezomib) and dexamethasone confirmed higher response rates, and improved progression free and overall survival (PFS/OS). Currently, these are the back bone of myeloma treatment.

\section{Treatment}

Current management of myeloma is based on the initial assessment for transplant eligibility. Patients who are $\leq 65$ to 70 years of age, in good ECOG performance status and without significant co-morbidities are considered transplant eligible. Such patients receive 3 to 6 cycles of induction therapy followed by ASCT followed by maintenance therapy. Goal of induction therapy is to reduce plasma cells burden and improve depth of response. Patients who are 'transplant ineligible' or elderly are advised induction therapy (9-12 cycles) followed by maintenance therapy. In addition all patients should receive supportive care in the form of bisphosphonates, initially 3 monthly for 1-2 years then at longer intervals (4).

\section{Induction Therapy}

Initial studies have used 2 drug-based induction in the form of thalidomide plus dexamethasone, lenalidomide plus dexamethasone or bortezomib plus dexamethasone. In last five years -3 drug combination - one immunomodulator (thalidomide or lenalidomide), one proteasome inhibitor (bortezomib) and dexamethasone are being used for induction. Three drug combinations are associated with higher response rate (complete and very good partial response), and better PFS and OS. Commonly used combinations include- bortezomib, thalidomide plus dexamethasone (VTD), bortezomib, lenalidomide plus dexamethasone (VRd), or bortezomib, cyclophosphamide plus dexamethasone(VCd) or bortezomib, liposomal doxorubicin plus dexamethasone (PAd). There is no head to head comparison between these combinations. A number of randomized trials (5-10) have confirmed high response rates (Table 1).

AIIMS Experience: In a randomized study (11), we compared lenalidomidedexamethasone $(n=97)$ versus thalidomidedexamethsone $(n=96)$. Response rate was $72.2 \%$ versus $68.7 \%, p=0.34$. At a median follow-up of 70 months, median overall survival was not reached in len-dexa arm versus 63 months in thal-dexa arm $(p=0.50)$. Subsequently, in 
Table 1: Novel agents based induction therapy prior to transplant (Adapted from ref 5 : Moreau et al, Blood 2015)

\begin{tabular}{|c|c|c|c|c|c|c|}
\hline \multirow[t]{2}{*}{$\begin{array}{l}\text { Study } \\
\text { (Ref) }\end{array}$} & \multirow[t]{2}{*}{ Treatment scheme } & \multirow{2}{*}{$\begin{array}{l}\text { No } \\
\text { of } \\
\text { Pts }\end{array}$} & \multicolumn{2}{|c|}{$\begin{array}{l}\text { Response rate } \\
(\%)\end{array}$} & \multirow{2}{*}{$\begin{array}{l}\text { Post } \\
\text { transplant } \\
\text { CR+ } \\
\text { VGPR } \\
(\%)\end{array}$} & \multirow[t]{2}{*}{ Long term outcome } \\
\hline & & & ORR & $\mathrm{CR}+\mathrm{VGPR}$ & & \\
\hline $\begin{array}{l}\text { IFM } \\
(5)\end{array}$ & $\begin{array}{l}\text { VADx4 } 4 \text { DCEP x2-ASCT } \\
\text { VDx4 } 4 \text { DCEP x } 2 \text {-ASCT }\end{array}$ & 242 & $\begin{array}{l}63 \\
78\end{array}$ & $\begin{array}{l}1+15 \\
6+38\end{array}$ & $\begin{array}{l}9+37 \\
16+54\end{array}$ & $\begin{array}{l}\text { PFS } 30 \text { months } \\
\text { PFS } 36 \text { months }\end{array}$ \\
\hline $\begin{array}{l}\text { GIMEMA } \\
\text { (6) }\end{array}$ & $\begin{array}{l}\text { VTDx3-ASCT-VTD x2- } \\
\text { TDx3-ASCT-TDx2-Dexa } \\
\text { maintenance }\end{array}$ & 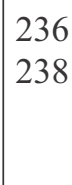 & $\begin{array}{l}93 \\
79\end{array}$ & $\begin{array}{l}\geq 62 \text { VGPR } \\
\geq 28 \text { VGPR }\end{array}$ & $\begin{array}{l}\geq 82 \text { VGPR } \\
\geq 64 \text { VGPR }\end{array}$ & $\begin{array}{l}\text { 3Yr PFS:68, } \\
\text { OS: } 86 \mathrm{mo} \\
3 \mathrm{Yr} \\
\text { PFS:56,OS:84mo }\end{array}$ \\
\hline $\begin{array}{l}\text { PETHEMA } \\
\text { (7) }\end{array}$ & $\begin{array}{l}\text { TDx6-ASCT-IFNm/Tm/VT } \\
\mathrm{mx} 3 \mathrm{Y} \\
\text { VTDx6-ASCT- } \\
\text { IFNm/Tm/VTmx 3Y } \\
\text { VBMCP/VBADx4-Vx2- } \\
\text { ASCT-IFNm/Tm/VTmx3Y }\end{array}$ & $\begin{array}{l}127 \\
130 \\
129\end{array}$ & $\begin{array}{l}62 \\
85 \\
75\end{array}$ & $\begin{array}{l}\geq 29 \text { VGPR } \\
\geq 60 \text { VGPR } \\
\geq 36 \text { VGPR }\end{array}$ & $\begin{array}{l}\text { CR: } 40 \\
\text { CR:57 } \\
\text { CR:48 }\end{array}$ & $\begin{array}{l}\text { PFS } 28 \text { mo, OS 65\% } \\
@ 4 \text { Yr } \\
\text { PFS:56 mo, OS 74\% } \\
\text { PFS:35 mo, OS:70\% }\end{array}$ \\
\hline IFM (8) & $\begin{array}{l}\text { VDx4-ASCT } \\
\text { VTDx4-ASCT }\end{array}$ & $\begin{array}{l}99 \\
100\end{array}$ & $\begin{array}{l}81 \\
88\end{array}$ & $\begin{array}{l}\geq 36 \text { VGPR } \\
49 \geq \text { VGPR }\end{array}$ & $\begin{array}{l}58 \geq \text { VGPR } \\
74 \geq \text { VGPR }\end{array}$ & $\begin{array}{l}\text { PFS : } 30 \mathrm{mo} \\
\text { PFS:26 mo }\end{array}$ \\
\hline $\begin{array}{l}\text { HOVON- } \\
65(9)\end{array}$ & $\begin{array}{l}\text { VADx3-CAD-ASCT- } \\
\text { Tmx2Y } \\
\text { PADx3-CAD-ASCT- } \\
\text { Vmx2Y }\end{array}$ & 414 & $\begin{array}{l}54 \\
78\end{array}$ & $\begin{array}{l}14 \geq \text { VGPR } \\
42 \geq \text { VGPR }\end{array}$ & $\begin{array}{l}36 \geq \text { VGPR } \\
62 \geq \text { VGPR }\end{array}$ & $\begin{array}{l}\text { PFS:28mo, OS 55\% } \\
\text { @,5 Yr } \\
\text { PFS:35 mo, OS 61\% } \\
\text { @,5 Yr }\end{array}$ \\
\hline
\end{tabular}

Abbreviations: CAD: cyclophosphamide-doxorubicin-dexamethasone; DCEP: dexamethasonecyclophosphamide-etoposide-cisplatin; Dm: dexamethasone maintenance; GIMEMA: Gruppo Italiano Malattie Ematologiche dell'Adulto; HOVON: Dutch-Belgian Hemato-Oncology Group; IFM: Intergroupe Francophone du Myélome; IFNm: interferon maintenance; NR: not reported; ORR: overall response rate; PAD: bortezomib-doxorubicin-dexamethasone; PETHEMA/GEM: Programa para el Estudio y la Terapéutica de las Hemopatías Malignas/Grupo Español de Mieloma; PR: partial response; TD: thalidomide-dexamethasone; Tm: thalidomide maintenance; V: bortezomib; VBAD: vincristine-BCNU-doxorubicin-dexamethasone; VBMCP: vincristine-BCNU-melphalancyclophosphamide-prednisone; Vm: bortezomib maintenance; VTD: bortezomib-thalidomidedexamethasone; VTm: bortezomib-thalidomide maintenance.

another study (12) a combination of bortezomiblenalidomide and dexamethasone (VRd) was compared to lenalidomide plus dexamethasone $(\mathrm{Rd})$. Overall response rates $(\mathrm{sCR}+\mathrm{CR}+\mathrm{VGPR}$ +PR) was $78.4 \%$ vs $73.9 \%$ in VRD and Rd arms, respectively, $\mathrm{p}=0.6$; sCR $+\mathrm{CR} 21(28.4 \%)$ and $21(30.4 \%)$, respectively, $\mathrm{p}=0.86$. At a median follow-up 17.1 months (range 1 to 33 ), median
OS is 30.2 months (95\% CI 28.2 to 32.2$)$ and 28.6 months $(95 \%$ CI 26 to 31.3$)$ in VRD and Rd arms, respectively, $\mathrm{p}=0.3$. Median PFS was 27.8 months (95\% CI 25.4 to 30.2$)$ and 28 months (95\% CI 24.6 to 31.4), respectively, $p=0.3$. Estimated one-year OS is $88 \%$ vs $85 \%$ in arms A and B, and PFS $83 \%$ vs $72 \%$, respectively (12). 
Table 2 :High dose chemotherapy and autologous stem cell transplantation with novel agents based induction therapy: Randomized trials

(adapted from ref. 17 Dhakal et al, 2018)

\begin{tabular}{|c|c|c|c|c|c|c|}
\hline $\begin{array}{l}\text { Author } \\
\text { (Ref) }\end{array}$ & $\begin{array}{l}\text { No of } \\
\text { Pts }\end{array}$ & Induction & $\begin{array}{l}\text { Conditioning } \\
\text { Vs } \\
\text { standard } \\
\text { therapy }\end{array}$ & Maintenance & $\begin{array}{l}\text { Follow-up } \\
\text { (in } \\
\text { months) }\end{array}$ & Comments \\
\hline $\begin{array}{l}\text { Palumbo } \\
\text { et al, } 2014 \\
\text { (13) }\end{array}$ & 273 & $\mathrm{Rd}$ & $\begin{array}{l}\text { Mel } 200 \mathrm{mg} \\
\mathrm{x} 2 \\
\text { Vs } \\
\text { MPR }\end{array}$ & $\begin{array}{l}\text { Len Vs } \\
\text { observation } \\
\text { until } \\
\text { progression }\end{array}$ & 51.2 & $\begin{array}{l}\text { Median PFS } 43 \text { mon } \\
\text { Vs } 22.4 \text { mon, } \\
\text { p }<0.001 \\
\text { OS @4 Yr } 81.6 \% \text { vs } \\
65.3 \%, p<0.02\end{array}$ \\
\hline $\begin{array}{l}\text { Gay et al, } \\
2015(14)\end{array}$ & 256 & $\mathrm{Rd}$ & $\begin{array}{l}\text { Mel } 200 \times 2 \\
\text { Vs CRd }\end{array}$ & $\begin{array}{l}\text { Len }+\mathrm{P} \text { vs } \\
\text { Len until } \\
\text { progression }\end{array}$ & 52 & $\begin{array}{l}\text { median PFS } 43.3 \mathrm{mo} \\
\text { vs } 28.6 \mathrm{mo}, \mathrm{p}<0.0001\end{array}$ \\
\hline $\begin{array}{l}\text { Attal et al, } \\
2015(15)\end{array}$ & 700 & RVd & $\begin{array}{l}\text { Mel 200x1 } \\
\text { Vs RVd x } 8 \\
\text { cycles }\end{array}$ & $\begin{array}{l}\text { Len for one } \\
\text { year }\end{array}$ & 44 & $\begin{array}{l}\text { Median PFS 50mo vs } \\
36 \text { mo,p }<0.001 \\
\text { OS @ } 4 \text { Yr } 81 \% \text { vs } \\
82 \%, p=\text { ns }\end{array}$ \\
\hline $\begin{array}{l}\text { Cavo et al, } \\
2016(16)\end{array}$ & 1192 & CyBord & $\begin{array}{l}\text { Mel200x } 1 \\
\text { or } 2 \\
\text { VsVMPx4 } \\
\text { cycles }\end{array}$ & $\begin{array}{l}\text { Len until } \\
\text { progression }\end{array}$ & 26 & $\begin{array}{l}\text { VGPR } 84 \% \text { vs } \\
74 \%, p<0.0001 \\
\text { PFS better with } \\
\text { HDCT, } p<0.01\end{array}$ \\
\hline
\end{tabular}

Rd: lenalidomide-dexamethsone; RVd: bortezomib, lenalidomide and dexamethsone; Len: lenalidomide; MPR: melphalan, lenalidomide- prednisolone, CRd: cyclophosphamide; lenalidomide: pdexamethasone; Mel: melphalan; VMP: bortezomib, melphalan, prednisolone; PFS: progression free survival; mo: months; HDCT: high dose chemotherapy.

\section{Autologous Stem Cell Transplantation (ASCT)}

Post induction therapy, transplant eligible patients undergo ASCT. A number of randomized studies have confirmed superiority of ASCT over conventional cytotoxic chemotherapy in these studies conducted before the year 2000. These studies confirmed superiority of high dose chemotherapy and stem cell transplant over conventional chemotherapy. With availability of novel agents from year 2000 onwards, four randomised studies (13-16) have been reported. Data from these studies have been summarized (17) in Table 2. High dose chemotherapy (HDCT) was associated with superior CR rates and improved PFS, confirming that even in novel agents era- HDCT followed by ASCT is the standard of care for transplant eligible myeloma patients.

\section{Procedure}

Prior to transplant all patients were evaluated for their fitness for transplant- for organ function, performance status and disease status. For peripheral blood stem cell mobilization patients receive inj G-CSF 10 $\mathrm{mcg} /$ day in 2 divided doses for 5 days followed by aphaeresis. Target is to collect $2-2.5 \times 10(6)$ CD34+ cells. About $10-20 \%$ patients may have poor mobilization. These can be identified by 
Table 3 : Maintenance therapy following ASCT : Phase 3 trials (Adapted from ref. 5 Moreau et al, Blood 2015)

\begin{tabular}{|c|c|c|c|c|c|c|}
\hline $\begin{array}{l}\text { Study } \\
\text { (Ref) }\end{array}$ & No of Pts & $\begin{array}{l}\text { Initial } \\
\text { Dose }\end{array}$ & $\begin{array}{l}\text { Response Vs } \\
\text { comparator }\end{array}$ & $\begin{array}{l}\text { Media } \\
\text { n } \\
\text { FU in } \\
\text { month } \\
\text { s }\end{array}$ & $\begin{array}{l}\text { EFS or PFS } \\
\text { Vs } \\
\text { Comparator }\end{array}$ & $\begin{array}{l}\text { OS Vs } \\
\text { comparator }\end{array}$ \\
\hline \multicolumn{7}{|l|}{ Thalidomide } \\
\hline Attal et al (18) & 597 & $400 \mathrm{mg}$ & $\begin{array}{l}\text { CR+VGPR } \\
67 \% \text { Vs } 55 \%\end{array}$ & 30mon & $\begin{array}{l}3 \text { Yr EFS } \\
52 \% \text { Vs } 36 \%\end{array}$ & $\begin{array}{l}87 \% \mathrm{Vs} 77 \% \\
\text { (a) } 4 \mathrm{Yr}\end{array}$ \\
\hline $\begin{array}{l}\text { Barlogie et al } \\
\text { (19) }\end{array}$ & 668 & $400 \mathrm{mg}$ & $\begin{array}{l}\text { CR: } 64 \% \text { Vs } \\
43 \%\end{array}$ & $72 \mathrm{mon}$ & $\begin{array}{l}\text { Median EFS } \\
6.0 \text { vs } 4.1 \mathrm{Yr}\end{array}$ & $\begin{array}{l}57 \% \mathrm{Vs} 44 \% \\
\text { @ } 8 \mathrm{Yr}\end{array}$ \\
\hline $\begin{array}{l}\text { Spencer et al } \\
(20)\end{array}$ & 269 & $200 \mathrm{mg}$ & $\begin{array}{l}\text { CR+VGPR } \\
63 \% \text { Vs } 40 \%\end{array}$ & $36 \mathrm{mon}$ & $\begin{array}{l}\mathrm{PFS} 42 \% \mathrm{Vs} \\
23 \% @ 3 \mathrm{Yr}\end{array}$ & $\begin{array}{l}86 \% \mathrm{Vs} 75 \% \\
\text { (a) } 3 \mathrm{Yr}\end{array}$ \\
\hline $\begin{array}{l}\text { Lokhorst et al } \\
\text { (21) }\end{array}$ & 556 & $50 \mathrm{mg}$ & $\begin{array}{l}\text { CR:31\% Vs } \\
23 \%\end{array}$ & $52 \mathrm{mon}$ & $\begin{array}{l}\text { Median PFS } \\
34 \text { Vs } 25 \\
\text { mon }\end{array}$ & $\begin{array}{l}\text { Median OS } \\
73 \text { Vs } 60 \\
\text { mon }\end{array}$ \\
\hline $\begin{array}{l}\text { Morgan et al } \\
(22)\end{array}$ & 492 & $50 \mathrm{mg}$ & NR & 38 mon & $\begin{array}{l}\text { Median PFS } \\
30 \text { Vs } 23 \\
\text { mon }\end{array}$ & $\begin{array}{l}75 \% \mathrm{Vs} 80 \% \\
\text { (a) } 3 \mathrm{yr}\end{array}$ \\
\hline $\begin{array}{l}\text { Steward et al } \\
(23)\end{array}$ & 332 & $200 \mathrm{mg}$ & NR & $4.1 \mathrm{Yr}$ & $\begin{array}{l}\text { PFS : 32\% } \\
\text { Vs 14\%@4 } \\
\text { Yr }\end{array}$ & $\begin{array}{l}68 \% \mathrm{Vs} 60 \% \\
\text { (a) } 4 \mathrm{Yr}\end{array}$ \\
\hline \multicolumn{7}{|l|}{ Lenalidomide } \\
\hline Attal et al (24) & 614 & $10 \mathrm{mg}$ & $\begin{array}{l}\text { CR+VGPR: } \\
84 \% \text { Vs } 76 \%\end{array}$ & 45 mon & $\begin{array}{l}\text { Median PFS: } \\
41 \mathrm{Vs} 23 \\
\text { mon }\end{array}$ & $\begin{array}{l}73 \% \mathrm{Vs} 75 \% \\
\text { (a) } 4 \mathrm{Yr}\end{array}$ \\
\hline $\begin{array}{l}\text { Mc Carthy et } \\
\text { al (25) }\end{array}$ & 460 & $10 \mathrm{mg}$ & NR & 34 mon & $\begin{array}{l}\text { Median TTP: } \\
46 \text { Vs } 27 \\
\text { mon }\end{array}$ & $\begin{array}{l}88 \% \mathrm{Vs} 80 \% \\
\text { (a) } 3 \mathrm{Yr}\end{array}$ \\
\hline \multicolumn{7}{|l|}{ Bortezomib } \\
\hline $\begin{array}{l}\text { Sonneveld et } \\
\text { al (10) }\end{array}$ & 827 & $1.3 \mathrm{mg} / \mathrm{m} 2$ & $\begin{array}{l}\text { CR+VGPR:7 } \\
6 \% \text { Vs } 56 \%\end{array}$ & 41 mon & $\begin{array}{l}\text { Median } \\
\text { PFS: } 35 \text { Vs } \\
28 \text { mon }\end{array}$ & $\begin{array}{l}61 \% \mathrm{Vs} 55 \% \\
\text { (a) } 5 \mathrm{Yr}\end{array}$ \\
\hline Rosinol (26) & 266 & $1.3 \mathrm{mg} / \mathrm{m} 2$ & NR & 24 mon & $\begin{array}{l}2 \text { Yr PFS } \\
78 \% \text { Vs } 63 \% \\
\text { Vs } 49 \%\end{array}$ & NR \\
\hline
\end{tabular}

CR: complete response; VGPR: very good partial response; EFS: event free survival; NR: not reported; PFS: progression free survival; OS: overall survival; TTP: time to progression. 
Table 4 : Induction therapy for transplant ineligible patients : Phase 3 studies and meta analysis results

(Adapted from ref. 5 Moreau et al, Blood 2015)

\begin{tabular}{|l|l|l|l|l|l|l|}
\hline $\begin{array}{l}\text { Study } \\
\text { (Ref) }\end{array}$ & Scheme & $\begin{array}{l}\text { No of } \\
\text { pts }\end{array}$ & $\begin{array}{l}\text { Median } \\
\text { FU }\end{array}$ & $\begin{array}{l}\text { Best } \\
\text { response }\end{array}$ & $\begin{array}{l}\text { PFS in } \\
\text { Months }\end{array}$ & $\begin{array}{l}\text { OS in } \\
\text { months }\end{array}$ \\
\hline $\begin{array}{l}\text { MPT meta } \\
\text { analysis } \\
(27)\end{array}$ & $\begin{array}{l}\text { MPTx8 vs 12 vs } \\
\text { until relapse }\end{array}$ & 1685 & $\begin{array}{l}\text { Not } \\
\text { available }\end{array}$ & VGPR25\% & 20.3 & 39.3 mo \\
\hline $\begin{array}{l}\text { MPT } \\
\text { First trial } \\
(28)\end{array}$ & MPTx12 cycles & 547 & 37 mon & CR9.3\% & 21.2 & $\begin{array}{l}51.4 \% @ 4 \\
\text { Yr }\end{array}$ \\
\hline CTD (29) & CTD -9 cycles & 426 & 44 & CR13.1\% & 13 & 33.2 \\
\hline $\begin{array}{l}\text { VMP (30) } \\
\text { VISTA } \\
\text { trial }\end{array}$ & VMP -9 cycles & 344 & 60.1 & CR30\% & 21.7 & 56.4 \\
\hline $\begin{array}{l}\text { MPR-R } \\
(31)\end{array}$ & $\begin{array}{l}\text { MPRx9 cycles } \\
\text { followed by R until } \\
\text { progression/relapse }\end{array}$ & 152 & 30 & CR9.9\% & 31 & $59 \% @ 4$ Yr \\
\hline $\begin{array}{l}\text { VMPT- } \\
\text { VT(32-33) }\end{array}$ & $\begin{array}{l}\text { VMPTx9 followed } \\
\text { by VTx 2 yrs or } \\
\text { until } \\
\text { progression/relapse }\end{array}$ & 254 & 54 & CR38\% & 35.3 & $61 \% @ 5$ Yr \\
\hline $\begin{array}{l}\text { VMP/VTP } \\
\text {-VT(34- } \\
\text { 35) }\end{array}$ & $\begin{array}{l}\text { VMP or VTPx6 } \\
\text { f/b VT up to 3 Yrs }\end{array}$ & 91 & 46 & CR46\% & 39 & $69 \% @ 5 Y r$ \\
\hline $\begin{array}{l}\text { Rd } \\
\text { continuous } \\
(36)\end{array}$ & $\begin{array}{l}\text { RD until disease } \\
\text { progression }\end{array}$ & 535 & 37 & CR15.1\% & 25.5 & $\begin{array}{l}59.4 \% @ 4 \\
\text { Yr }\end{array}$ \\
\hline
\end{tabular}

Abbreviations: CAD: cyclophosphamide-doxorubicin-dexamethasone; DCEP: dexamethasonecyclophosphamide-etoposide-cisplatin; Dm: dexamethasone maintenance; GIMEMA: Gruppo Italiano Malattie Ematologiche dell'Adulto; HOVON: Dutch-Belgian Hemato-Oncology Group; IFM: Intergroupe Francophone du Myélome; IFNm: interferon maintenance; NR: not reported; ORR: overall response rate; PAD: bortezomib-doxorubicin-dexamethasone; PETHEMA/GEM: Programa para el Estudio y la Terapéutica de las Hemopatías Malignas/Grupo Español de Mieloma; PR: partial response; TD: thalidomide-dexamethasone; Tm: thalidomide maintenance; V: bortezomib; VBAD: vincristine-BCNU-doxorubicin-dexamethasone; VBMCP: vincristine-BCNU-melphalancyclophosphamide-prednisone; Vm: bortezomib maintenance; VTD: bortezomib-thalidomidedexamethasone; VTm: bortezomib-thalidomide maintenance. 
doing peripheral blood CD34 counts on day 4 of G-CSF. Patients with CD34 + cells $<20 / \mathrm{cmm}$ are likely to have poor mobilization. Options for such patients include- chemo-mobilization using cyclophosphamide $2-4 \mathrm{~g} / \mathrm{m}^{2}$ or Plerixafor, a CXCR4 -chemokine nhibitor. Patients with prior melphalan or radiation are poor mobilizers and therefore these should be avoided during induction in transplant eligible patients. Once adequate number of stem cells are harvested these can be cryopreserved at - 80 degree Cels or in liquid nitrogen for long term storage. At our centre, we collect stem cells electively, keep at 4 degree Cel. This is followed by high dose chemotherapy with melphalan $200 \mathrm{mg} / \mathrm{m}^{2}$ IV followed by stem cell infusion 24 hours later. This practice of keeping stem cells at 4 degree is cost effective and stem cells are viable $(>90 \%)$ up to 96 hours. Twenty four hours after stem cells, patients are started on G-CSF $5 \mathrm{mcg} / \mathrm{kg}$ once daily until engraftment. Once stable, patients are then discharged and followed-up in the out patients department with reassessment for response on day 100. Our current policy is to give 2 more cycles of $\mathrm{VRd}$ regimen as consolidation from day 100 ( \pm 7 days) onwards followed by maintenance therapy using lenalidomide $10 \mathrm{mg}$ daily for 21 days every 28 days for 2 years. Patients intolerant to lenalidomide receive inj bortezomib $2 \mathrm{mg}$ subcutaneously every 2 weeks. In earlier period we have used low dose thalidomide $(50 \mathrm{mg}$ daily).

\section{Post transplant consolidation}

A number of studies have suggested that 2-3 cycles of consolidation using VRd or VTd may further improve $\mathrm{CR}$ rate and more patients have 'nil' minimal residual disease. In an ongoing prospective study at our centre among 58 patients CR rate improved from $81.3 \%$ (post ASCT at day 100) to $89.8 \%$ post consolidation, 32 of 58 were MRD negative at day $+100,26$ were MRD +ve, of these $16(61.5 \%)$ became MRD negative post-consolidation as assessed by 8 colour flow cytometry) (unpublished data).

\section{Maintenance therapy}

Initial studies have used thalidomide 100 to $200 \mathrm{mg}$ per day; among six randomized studies (18-23) 3 had shown improved PFS and OS. Neuropathy was the main toxicity. Subsequent studies have used lenalidomide 5-10 mg daily 15-21 days every 28 days. These studies have shown improved PFS. Second malignancy has been reported in $4-6 \%$ of patients. Inj Bortezomib $2 \mathrm{mg}$ every two weeks has been used in studies from Europe. Currently, Lenalidomide $10 \mathrm{mg}$ daily for 21 days out of 28 days for two years is recommended (Table 3 ).

\section{Induction therapy for transplant ineligible or elderly patients}

Initial studies confirmed superiority of melphalan- thalidomide and prednisolone (MPT) combination over MP alone as regards to response rate, PFS and OS (Table 4). VISTA trial compared VMP (bortezomib, melphalan and prednisolone) with MP; VMP was superior in terms of response rate, $\mathrm{CR}$ rate, median time to progression (24.4 months vs. 16.6 months) and OS. In a recent update at 60 months, these results still hold; median OS 56 months versus 43 months (32-33). Recent studies have used a combination of MP-lenalidomide (MPR) (31). Another phase 3 study has compared lenalidomide plus dexamethasone (Ld) as continuous therapy or 18 cycles to MPT. At a median follow-up of 37 months; continuous Ld was better compared to Ld 18 cycles and MPT regimen (36). For patients who are frail or have significant co-morbidities- two drug combinations is a reasonable choice.

\section{Conclusions}

Survival of patients with myeloma has improved significantly in the past two decades. Compared to median survival of 3-to 3.5 years prior to year 2000, presently median survival is 5.5 to 6 years. For patients undergoing ASCT median survival is 8 to 9 years. Recently a number of newer agents have been added with 
significant activity and have been approved for the treatment of relapse. These includecarfilzomib, ixazomib, pomalidomide. In addition, two monoclonal antibodies- daratumab -anti CD38 and elotuzumab (anti SLAM F7) have been moved to front line. These are now being compared in combination with bortezomib, carfilzomib, or lenalidomide based combinations. Other strategies currently being explored include- vaccination against MM antigens, along with immunomodulatory agents such as IMiDs or the anti-PD-1 antibody and CAR-T cell therapy. It is hoped that these strategies would lead to further improvement in response and long-term control of the disease in near future.

\section{References}

1. India State-Level Disease Burden Initiative Cancer Collaborators (2018). The burden of cancers and their variations across the states of India: the Global Burden of Disease Study 1990-2016. Lancet Oncol 19(10):1289-1306.

2. Kumar L, Verma R, Radhakrishnan VR (2010). Recent advances in the management of multiple myeloma. Natl Med J India 23(4): 210-218.

3. Kumar SK, Rajkumar SV, Dispenzieri A, et al (2008). Improved survival in multiple myeloma and the impact of novel therapies. Blood 111(5): 2516-2520.

4. Richter J, Jagannath S (2018). Society of Hematologic Oncology State of the Art Update and Next Questions: Multiple Myeloma. Clin Lymphoma Myeloma Leuk 18(11): 693-702.

5. Moreau P, Attal M, Facon T (2015). Frontline therapy of multiple myeloma. Blood 125: 3076-3084.

6. Harousseau JL, Attal M, Avet-Loiseau H, et al (2 010$)$. Bortezomib plus dexamethasone is superior to vincristine plus doxorubicin plus dexamethasone as induction treatment prior to autologous stem-cell transplantation in newly diagnosed multiple myeloma: results of the IFM 2005-01 phase III trial. J Clin Oncol 28(30): 4621-4629.

7. Cavo M, Tacchetti P, Patriarca F, et al; GIMEMA Italian Myeloma Network (2010). Bortezomib with thalidomide plus dexamethasone compared with thalidomide plus dexamethasone as induction therapy before, and consolidation therapy after, double autologous stem-cell transplantation in newly diagnosed multiple myeloma: a randomised phase 3 study. Lancet 376: 2075-2085.

8. Rosiñol L, Oriol A, Teruel AI, et al; Programa para el Estudio y la Terapéutica de las Hemopatías Malignas/Grupo Español de Mieloma (PETHEMA/GEM) group (2012). Superiority of bortezomib, thalidomide, and dexamethasone (VTD) as induction pretransplantation therapy in multiple myeloma: a randomized phase 3 PETHEMA/GEM study. Blood 120(8):1589-1596.

9. Moreau P, Avet-Loiseau H, Facon T, et al (2011). Bortezomib plus dexamethasone versus reduced-dose bortezomib, thalidomide plus dexamethasone as induction treatment before autologous stem cell transplantation in newly diagnosed multiple myeloma. Blood 118: 5752-5758.

10. Sonneveld P, Schmidt-Wolf IG, van der Holt B, et al (2012). Bortezomib induction and maintenance treatment in patients with newly diagnosed multiple myeloma: results of the randomized phase III HOVON-65/GMMG-HD4 trial. J Clin Oncol30(24): 2946-2955.

11. Kumar L, Mookerjee A, Sharma A, Gupta R, Sharma OD, Srinivas V (2015). Low dose dexamethasone plus lenalidomide (Len-dexa) versus thalidomide (Thal-dexa) as induction therapy for newly diagnosed patients of multiple myeloma: a phase III, randomized study. Clin Lymphoma Myeloma Leuk 15(suppl3): e146. 
12. Mookerjee A, Gupta R, Jasrotia S, et al (2017). Bortezomib, Lenalidomide and Low-Dose Dexamethasone (VRD) Versus Lenalidomide and Low-Dose Dexamethasone (Ld) for NewlyDiagnosed Multiple Myeloma- a Randomized Phase III Study. Blood 130(suppl 1): 906.

13. Palumbo A, Cavallo F, Gay F, et al (2014). Autologous transplantation and maintenance therapy in multiple myeloma. NEngl J Med 371(10): 895-905.

14. Gay F, Oliva S, Petrucci MT, et al (2015). Chemotherapy plus lenalidomide versus autolo gous transplantation, followed by lenalidomide plus prednisone versus lenalidomide maintenance, in patients with multiple myeloma: a randomized, multicentre, phase 3 trial. Lancet Oncol 16: 1617-1629.

15. Attal M, Lauwers-Cances V, Hulin C, et al; IFM 2009 Study (2017). Lenalidomide, bortezomib, and dexamethasone with transplantation for myeloma. $N$ Engl J Med 376: 1311-1320.

16. Cavo M, Palumbo A, Zweegman S, et al (2016). Upfront autologous stem cell transplantation (ASCT) versus novel agent-based therapy for multiple myeloma (MM): a randomized phase 3 study of the European Myeloma Network (EMN02/HO95 MM trial). J Clin Oncol 34: 15(suppl).

17. Dhakal B, Szabo A, Chhabra S, et al (2018). Autologous transplantation for newly diagnosed multiple myeloma in the era of novel agent induction: a systematic review and meta-analysis. JAMA Oncol 4(3): 343350 .

18. Attal M, Harousseau JL, Leyvraz S, et al; Inter-Groupe Francophone du Myélome (IFM) (2006). Maintenance therapy with thalidomide improves survival in multiple myeloma patients. Blood 108: 3289-3294.

19. Barlogie B, Pineda-Roman M, van Rhee F, et al (2008). Thalidomide arm of Total Therapy 2 improves complete remission duration and survival in myeloma patients with metaphase cytogenetic abnormalities. Blood 112(8): 3115-3121.

20. Spencer A, Prince HM, Roberts AW, et al (2009). Consolidation therapy with lowdose thalidomide and prednisolone prolongs the survival of multiple myeloma patients undergoing a single autologous stem-cell transplantation procedure. JClin Oncol 27: 1788-1793.

21. Lokhorst HM, van der Holt B, Zweegman $\mathrm{S}$, et al; Dutch-Belgian Hemato-Oncology Group (HOVON) (2010). A randomized phase 3 study on the effect of thalidomide combined with adriamycin, dexamethasone, and high-dose melphalan, followed by thalidomide maintenance in patients with multiple myeloma. Blood 115:1113-1120.

22. Morgan GJ, Gregory WM, Davies FE, et al; National Cancer Research Institute Haematological Oncology Clinical Studies Group (2012). The role of maintenance thalidomide therapy in multiple myeloma: MRC Myeloma IX results and metaanalysis. Blood 119(1): 7-15.

23. Stewart AK, Trudel S, Bahlis NJ, et al (2013). A randomized phase 3 trial of thalidomide and prednisone as maintenance therapy after ASCT in patients with MM with a quality-of-life assessment: the National Cancer Institute of Canada Clinicals Trials Group Myeloma 10 Trial. Blood 121: 1517-1523.

24. Attal M, Lauwers-Cances V, Marit G, et al; IFM Investigators (2012). Lenalidomide maintenance after stem-cell transplantation for multiple myeloma. $N$ Engl J Med 366: 1782-1791.

25. McCarthy PL, Owzar K, Hofmeister CC, et al (2012). Lenalidomide after stem-cell transplantation for multiple myeloma. $N$ Engl J Med 366(19): 1770-1781. 
26. Rosiñnol L, Oriol A, Teruel AI, et al (2012). Maintenance therapy after stem-cell transplantation for multiple myeloma with bortezomib/thalidomide vs thalidomide vs alfa2b-interferon: final results of a phase 3 PETHEMA/GEM randomized trial. ASH annual meeting abstracts (Abstract). Blood 120(21). Abstract 334 .

27. Fayers PM, Palumbo A, Hulin C, et al; Nordic Myeloma Study Group; Italian Multiple Myeloma Network; Turkish Myeloma Study Group; HematoOncologie voor Volwassenen Nederland; Intergroupe Francophone du Myélome; European Myeloma Network (2011). Thalidomide for previously untreated elderly patients with multiple myeloma: meta-analysis of 1685 individual patient data from 6 randomized clinical trials. Blood 118(5): 1239-1247.

28. Facon T,Mary JY, Hulin C, et al; Intergroupe Francophone du Myélome (2007). Melphalan and prednisone plus thalidomide versus melphalan and prednisone alone or reduced-intensity autologous stem cell transplantation in elderly patients with multiple myeloma (IFM 99-06): a randomised trial. Lancet 370(9594): 1209-1218.

29. Morgan GJ, Davies FE, Gregory WM, et al; NCRI Haematological Oncology Study Group (2011). Cyclophosphamide, thalidomide, and dexamethasone (CTD) as initial therapy for patients with multiple myeloma unsuitable for autologous transplantation. Blood 118(5): 1231-1238.

30. San Miguel JF, Schlag R, Khuageva NK, et al; VISTA Trial Investigators (2008). Borte zomib plus melphalan and prednisone for initial treatment of multiple myeloma. $N$ Engl J Med 359(9): 906-917.

31. Palumbo A, Hajek R, Delforge M, et al;
MM-015 Investigators (2012). Continuous lenalidomide treatment for newly diagnosed multiple myeloma. $N$ Engl J Med366(19): 1759-1769.

32. Palumbo A, Bringhen S, Rossi D, et al (2010). Bortezomib-melphalanprednisone-thalidomide followed by maintenance with bortezomib-thalidomide compared with bortezomib-melphalanprednisone for initial treatment of multiple myeloma: a randomized controlled trial. $J$ Clin Oncol 28(34): 5101-5109.

33. Palumbo A, Bringhen S, Larocca A, et al (20 014 ). Bortezomib-melphalanprednisone-thalidomide followed by maintenance with bortezomib-thalidomide compared with bortezomib-melphalanprednisone for initial treatment of multiple myeloma: updated follow-up and improved survival. JClin Oncol 32(7): 634-640.

34. Mateos MV, Oriol A, Martínez-López J, et al (2010). Bortezomib, melphalan, and prednisone versus bortezomib, thalidomide, and prednisone as induction therapy followed by maintenance treatment with bortezomib and thalidomide versus bortezomib and prednisone in elderly patients with untreated multiple myeloma: a randomised trial. Lancet Oncol 11(10): 934-941.

35. Mateos MV, Oriol A, Martínez-López J, et al (2012). Maintenance therapy with bortezomib plus thalidomide or bortezomib plus prednisone in elderly multiple myeloma patients included in the GEM2005MAS65 trial. Blood 120(13): 2581-2588.

36. Benboubker L, Dimopoulos MA, Dispenzieri A, et al; FIRST Trial Team (2014). Lenalidomide and dexamethasone in transplant-ineligible patients with myeloma. NEngl J Med371(10):906-917. 